\title{
DIPTERES PEU CONNUS DES SEDIMENTS D'EAU COURANTE : I. - LES PTYCHOPTERIDAE (NYMPHOSE ET ACCOUPLEMENT) DU SUD-OUEST DE LA FRANCE

\author{
[NEMATOCERA]
}

par A. G. B. THOMAS 1

Les nymphes $\hat{\delta}$ et $q$ des trois espèces rencontrées en eau courante dans le Sud-Ouest de la France (Ptychoptera albimana, $P$. lacustris et $P$. paludosa) font l'objet d'une clé de détermination. A des températures constantes comprises entre 25 et $6^{\circ} \mathrm{C}$, la durée de la nymphose de $P$. albimana varie de 6 à 31 jours. Entre 25 et $15^{\circ} \mathrm{C}$, la nymphose de $P$. lacustris a la même durée que celle de $P$. albimana, mais à $10^{\circ} \mathrm{C}$, elle est plus courte d'une semaine. Les variations de température ont une action immédiate sur l'apparition des nouvelles nymphes.

La longévité des imagos $\hat{f}$ est supérieure à celle des $ᄋ$. Il a été observé jusqu'à 5 accouplements successifs sur un même couple. La durée de l'accouplement diminue avec une élévation de la température. Dans la nature, les $q$ sont souvent fécondées dès leur émergence. En début de période de vol, les $\hat{\imath}$ apparaissent probablement les premiers. La ponte suit en général l'accouplement de moins de 24 heures.

\section{Poorly-known Diptera from the sediments of flowing water : \\ I. The Ptychopteridae (pupation and mating) from South-West of France (Nematocera).}

A key is given to the pupae of males and females of three species found in South-West France (Ptychoptera albimana, P. lacustris, P. paludosa). At constant temperatures between 25 and $6^{\circ} \mathrm{C}$, the length of the pupation period for $P$. albimana varies from $6-31$ days. Between 25 and $15{ }^{\circ} \mathrm{C}$, the length of the pupation period for $P$. lacustris is similar to that for $P$. albimana, but it is shorter a week at $10^{\circ} \mathrm{C}$. Variations in temperature have an immediate effect on the appearance of new pupae.

The life-span of male imagines is longer than that of females. Up to five successive matings have been observed in the same couple. The duration of mating decreases with an increase in temperature. In nature, the females are often fecund when they emerge. The males probably appear first at the start of the flight period. The period between mating and oviposition is usually less than 24 hours.

1. Laboratoire d'Hydrobiologie, Université Paul-Sabatier, 118, route de Narbonne, 31077 Toulouse Cedex, France. 
La famille des Ptychopteridae ou Liriopeidae, à peu près exclusivement aquatique, est l'une des plus ignorées des hydrobiologistes. Cependant, on sait depuis fort longtemps que les larves possèdent un tube respiratoire postérieur plus ou moins long qui leur permet de coloniser des sédiments fins, riches en matière organique (mares, étangs...). La taxonomie des imagos des espèces européennes est par ailleurs très au point. Il est donc d'autant plus regrettable que les citations de larves de Ptychopteridae soient aussi rares dans les études de benthos d'eau courante où cette famille demeure à peu près inconnue.

La présente étude est limitée aux trois espèces que j'ai rencontrées en eau courante jusqu'ici : Ptychoptera albimana (Fabricius, 1787), $P$. lacustris Meigen, 1830 et $P$. paludosa Meigen, 1804. Les deux premières sont plus particulièrement localisées près des sources limnocrènes (crénon). Toutes trois vivent dans les sédiments immergés - P. lacustris parfois dans les graviers - en courant lent, de vitesse en général inférieure à $20 \mathrm{~cm} / \mathrm{s}$ et font partie de l'hydropsammon (Angelier 1953). Les imagos des trois espèces se rencontrent d'ailleurs au voisinage immédiat des cours d'eau (Tonnoir 1919, Thomas 1968 et 1977, Stubbs 1972, etc.) quoiqu'en faible proportion comparativement aux autres familles de Diptères aquatiques ou très hygrophiles (Thomas, 1969).

Le cycle biologique des Ptychopteridae est fort mal connu, en particulier - comme d'ailleurs chez beaucoup d'insectes aquatiques - au niveau des nymphes et de l'incubation des œufs. Cette première note traite exclusivement de la nymphose et des modalités de l'accouplement. En raison du nombre de spécimens nécessaire (larves âgées, nymphes et imagos), ce travail a surtout été axé sur l'espèce la plus susceptible de pulluler localement : $P$. albimana, dont environ 300 nymphes $\hat{o}$ et 160 nymphes $q$ ont été obtenues par élevage de larves.

M. C. Torossian qui assure l'enseignement de Photographie scientifique à l'Université de Toulouse s'est chargé des photographies ci-incluses et j'ai plaisir à l'en remercier.

\section{Diagnose des imagos}

L'aspect des Ptychopteridae rappelle celui des Tipulidae s. s., d'où leur nom de "false Crane Flies». Mais leur taille est en général nettement inférieure et leurs pattes plus robustes. Comme chez tous nos Nématocères, l'aile est caractéristique et permet une séparation aisée des autres familles lors du tri des chasses globales. Cette aile a la particularité de posséder des venae spuriae dont la plus visible est située entre les nervures $\mathrm{R} 4+5$ et $M 1+2$, à peu près à milongueur de la transverse rm. Sur tous les spécimens de $P$, albimana 
que j'ai pu examiner, il s'agit d'une formation nettement individualisée, un peu moins épaisse que R $4+5$ ou $M 1+2$ (photo 1 ), ce qui est en accord avec la figure 36 de Peus (1958). Chez P. paludosa, c'est un simple pli dont la crête est légèrement épaissie. Chez $P$. lacustris, la vena spuria est pratiquement inexistante, réduite à un pli.

La détermination spécifique des imagos obtenues par élevage a été effectuée avec les travaux de Freeman (1950) et Zitek-Zwyrtek (1971).

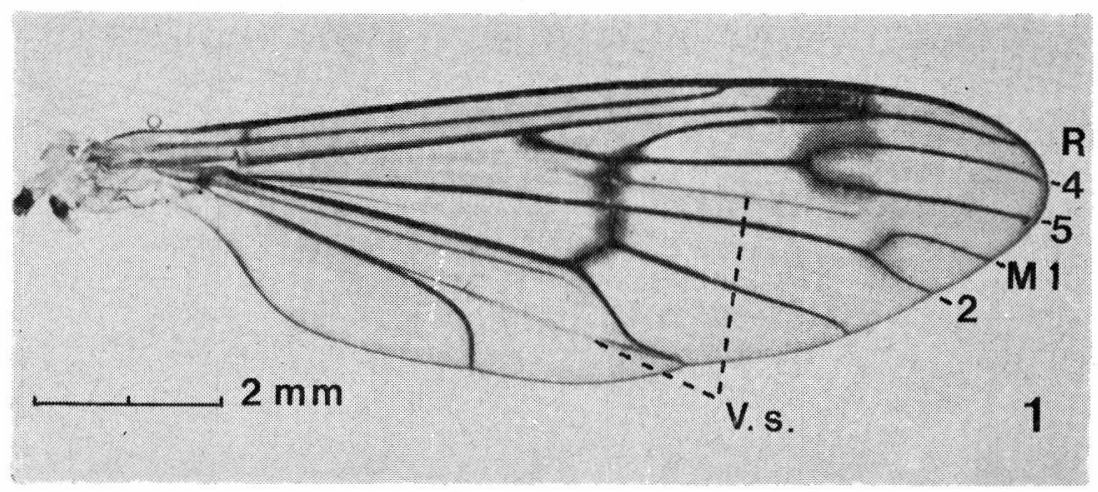

Рното 1. - Aile de Ptychoptera albimana. V. s. : vena spuria.

\section{I. - LA NYMPHOSE}

\section{Détermination spécifique des nymphes}

Les nymphes de Ptychopteridae ont été l'objet, dans les manuels d'hydrobiologie en particulier, de nombreuses descriptions pour la plupart sommaires et inutilisables au niveau spécifique. En Europe, quelques références peuvent cependant être retenues: Topsent (1916) sur $P$. albimana, Seligo (1928) sur $P$. contaminata et $P$. paludosa, Joost (1974) sur $P$. albimana et $P$. peusi.

Les principaux caractères morphologiques distinctifs de $P$. albimana, $P$. lacustris et $P$. paludosa sont présentés ci-dessous sous forme de clé de détermination pour les deux sexes. On trouvera dans Peus (1958) une description détaillée de la morphologie typique des nymphes de Ptychopteridae.

1. Segments abdominaux portant sur les pleures 3 rangées longitudinales de tubercules épineux (fig. 10) parfois partiellement dichotomisées par endroits en 4 ou 5 rangées (partie postérieure des segments). Dans l'ensemble, ornementation épineuse de tout l'abdomen moins nettement apparente que chez les deux autres espèces mais corps d'aspect plus velu (fig. 11). Chez les $\hat{o}$, contour apical du dernier segment assez saillant et fortement entaillé dans 
le plan de symétrie ; 2 paires de prolongements latéraux coniques bien visibles (fig. 2) ; bord postérieur médian du $3^{\mathrm{e}}$ sternite abdominal portant une forte excroissance en forme de double poche saillante non épineuse (fig. 13) ....... lacustris Meigen, 1830.

- Segments abdominaux portant sur les pleures 5 rangées longitudinales de tubercules épineux (fig. 9) parfois plus ou moins conver. gentes par endroits. Chez les $\hat{\delta}, 1$ ou 2 paires de prolongements latéraux sur le dernier segment abdominal; bord postérieur médian du $3^{\mathrm{e}}$ sternite abdominal avec ou sans une telle excroissance .................................... 2 .

2. Tubercules épineux des tergites abdominaux portés par des épaississements chitineux transversaux étroits et le plus souvent continus d'une pleure à l'autre, d'où un aspect nettement strié (fig. 7).
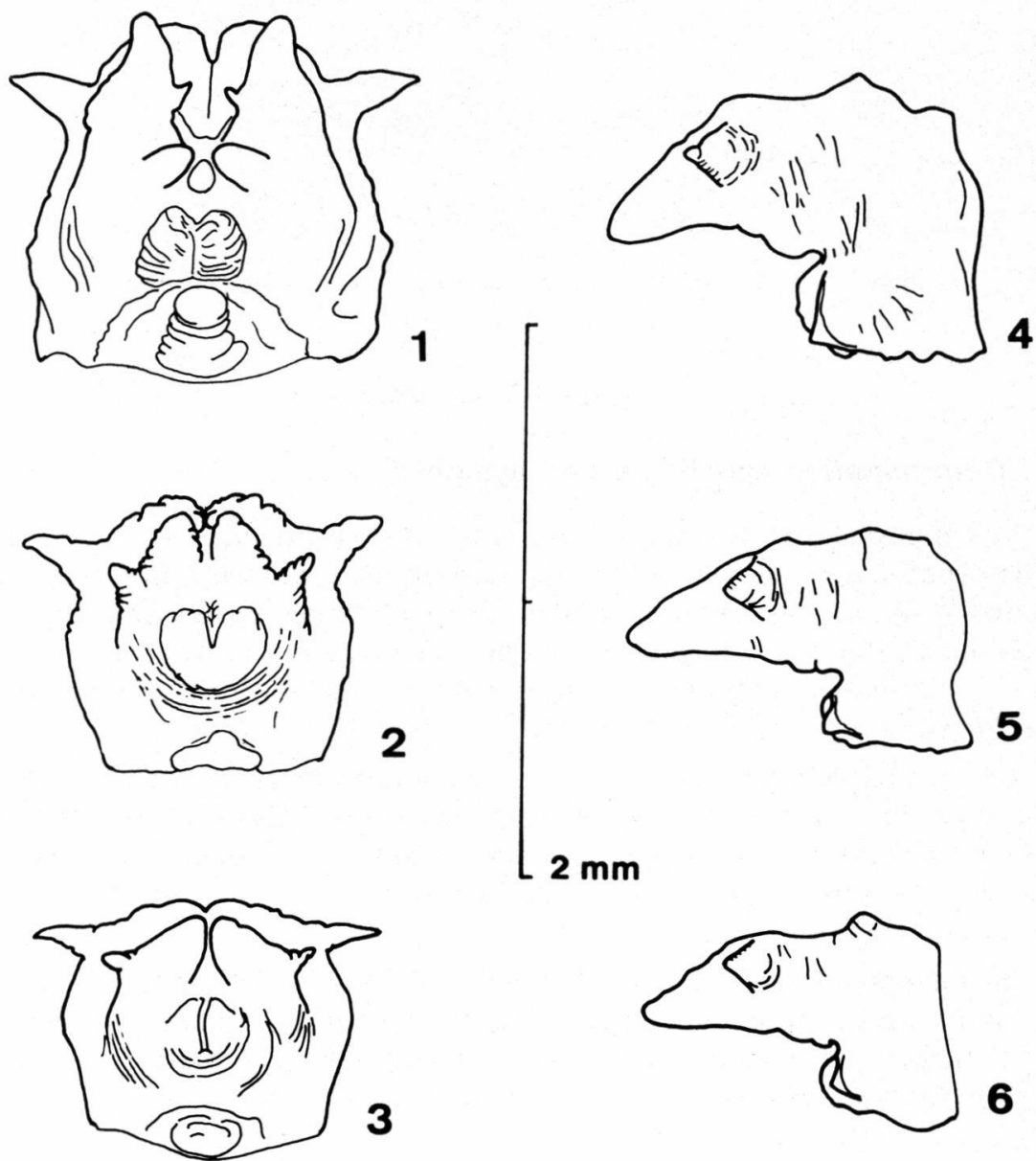

Fic. 1 à 6. - Terminalia $\hat{o}$ (à gauche) et 9 (à droite) de Ptychoptera spp. : albimana $(1,4)$, lacustris $(2,5)$ et paludosa $(3,6)$. 
Chez les o, 2 paires de prolongements latéraux sur le dernier segment abdominal et contour apical de ce dernier peu convexe et peu entaillé dans le plan de symétrie ; forte excroissance non épineuse sur le bord postérieur médian du $3^{\mathrm{e}}$ sternite abdominal .... $\ldots \ldots \ldots \ldots \ldots \ldots \ldots \ldots \ldots \ldots \ldots \ldots \ldots \ldots$ paludosa Meigen, 1804.

— Tubercules épineux des tergites abdominaux portés par des renforts chitineux transversaux interrompus plusieurs fois d'une pleure à l'autre, d'où un aspect assez peu strié (fig. 8). Épines
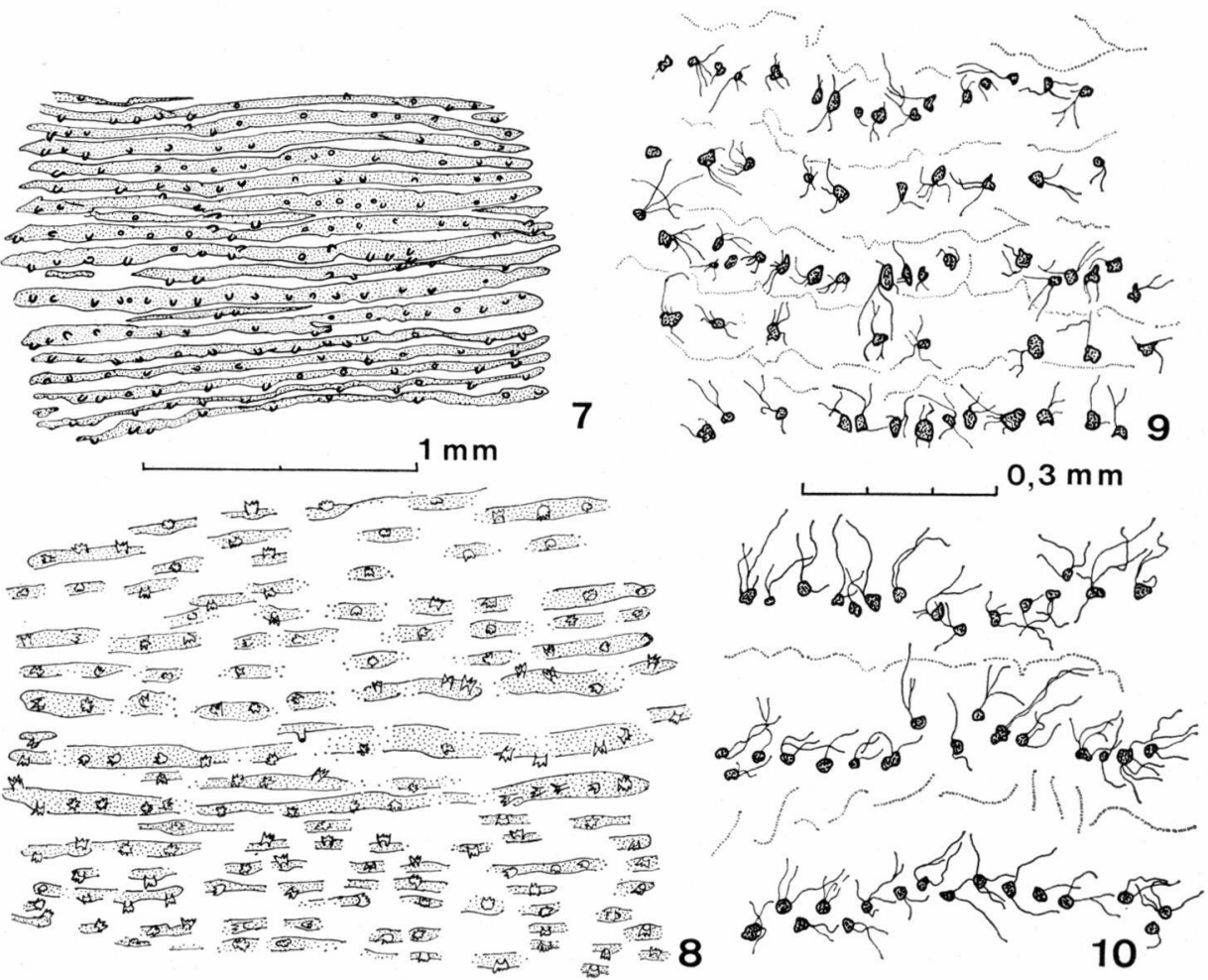

Fig. 7 à 10. - Ornementations cuticulaires sur le $4^{\text {e }}$ segment abdominal de Ptychoptera spp.:

1) à gauche, tergites de $P$. paludosa (7) et de $P$. albimana (8). Sur la fig. 8 , le quart antérieur environ du tergite n'a pas été représenté.

2) à droite, région médiane de pleures de $P$. paludosa (9) et de P. lacustris (10).

nettement disposées en couronnes portées par des excroissances basales plus hautes que chez les deux autres espèces (fig. 12) : l'abdomen paraît ainsi plus fortement épineux. Chez les $\hat{\delta}, 1$ paire seulement de prolongements latéraux et 1 paire de prolongements longitudinaux sur le dernier segment abdominal (fig. 1); aucune excroissance médiane sur le bord postérieur du $3^{\mathrm{e}}$ sternite abdo- 
minal : à cet endroit, la rangée transversale de tubercules épineux est seulement atrophiée par rapport à celles des segments 4 et 5 . En vue latérale, terminalia des $\$$ plus épais et angle de l'arête dorsale au niveau des prolongements latéraux plus marqué que chez les deux autres espèces (fig. 4) ....... albimana (Fabricius, 1787).
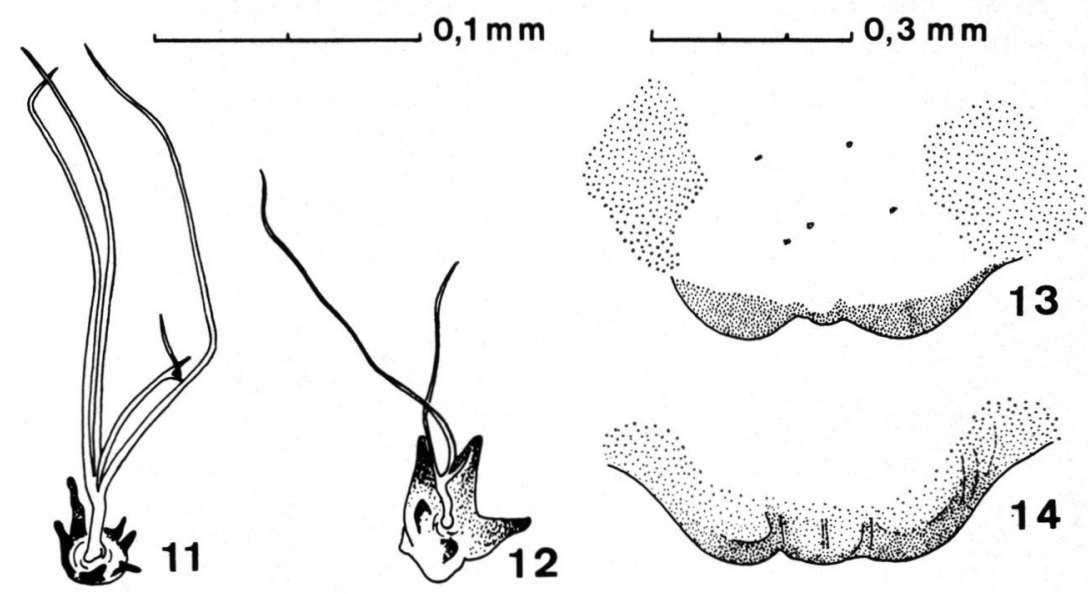

Fig. 11 à 14 . - Soies ramifiées et tubercules épineux du $4^{\mathrm{e}}$ tergite abdominal de $P$. lacustris (11) et de P. albimana (12). Excroissance médiane du bord postérieur du $3^{\circ}$ sternite abdominal de $P$. lacustris (13) et de $P$. paludosa (14).

\section{Durée}

La mesure de la durée de la nymphose implique une appréciation précise des passages respectifs de la larve à la nymphe puis de la nymphe à l'adulte. Cette estimation est, dans le cas présent, rendue malaisée du fait du décalage de temps qui existe entre le début des transformations anatomiques et histologiques que subit progressivement la larve - tout en gardant cependant sa morphologie externe - et l'apparition de la "nymphe » proprement dite, apparition qui n'a lieu qu'à l'exuviation. Selon Ameen (1969), ce délai serait d'un peu plus de 4 jours vers $19^{\circ} \mathrm{C}$. A ce sujet d'ailleurs, l'unanimité des auteurs est loin d'être faite pour fixer le début de l'état nymphal (Ameen, t. c.).

La " nymphose » a été considérée ici d'exuviation à exuviation, c'està-dire de la libération de la nymphe à celle de l'adulte - ce qui correspond donc à "the non pharate pupa plus the pharate adult " d'Ameen - pour les raisons suivantes: commodité de détermination des différents états à l'œil nu, et, par là même, suppression dans les élevages de longue durée des manipulations et examens répétés des 
larves vivantes sous loupe binoculaire et réduction dans de grandes proportions des risques de lésions accidentelles.

D'autre part, les répercussions de la nymphose d'une espèce sur le fonctionnement d'un écosystème sont essentiellement engendrées par les changements de biotope ou de comportement, face à un prédateur par exemple, qu'elle entraîne chez cette espèce. Or, chez les Ptychopteridae, la mobilité de la larve n'est en réalité profondément perturbée que quelques heures avant l'exuviation.

Mode opératoire

La durée de la nymphose a été déterminée à partir de larves âgées prélevées pendant la période de vol ou au début de celle-ci. Ces larves ont été maintenues dans le sédiment de leur biotope naturel à différentes températures constantes. Un tri de ce sédiment a été effectué sur tamis régulièrement, à de courts intervalles de temps $(12 \mathrm{~h})$ dans le but d'isoler les nymphes nouvellement formées. Celles-ci se distinguent très facilement à l'œil nu. En effet, après le rejet de l'exuvie larvaire, assez lent et effectué d'avant en arrière (photo 2), le corps de la jeune nymphe apparaît entièrement blanc pendant les premières heures. La tête et le thorax foncent ensuite rapidement, prenant d'abord une teinte cuivrée $\left(6 \mathrm{~h}\right.$ après environ, vers $\left.15^{\circ} \mathrm{C}\right)$ puis brun clair et enfin brun noirâtre. Le tube respiratoire postérieur disparaît avec l'exuvie larvaire tandis qu'apparaît dès le début - elle existe déjà, pelotonnée, dans la larve âgée avant l'exuviation - la corne respiratoire thoracique droite, antérieure, plus fine, plus rigide et plus longue (photo 3). Cette dernière est terminée par une palette préhensile capable d'assurer l'accrochage de la nymphe sur un relief émergé fixe (plante herbacée, etc.). En effet, contrairement à celle des Tipulidae, la nymphose n'est pas directement aćrienne: la nymphe demeure dans le même milieu que la larve, d'où elle respire grâce à sa corne thoracique droite ${ }^{1}$.

Les jeunes nymphes ont ensuite été isolées dans des piluliers à couvercle perforé contenant quelques $\mathrm{cm}^{3}$ de sédiment saturé d'eau, et élevées à température constante jusqu'à l'émergence des imagos.

Caractéristiques moyennes du sédiment utilisé (lors d'un prélèvement effectué le 13 octobre) :

- teneur en eau : $853 \%$ du poids sec $\left(24 \mathrm{~h}\right.$ à $\left.90^{\circ} \mathrm{C}\right)$.

- teneur en matière organique: plus de $50 \%$ du poids sec $\left(2 \mathrm{~h}\right.$ à $\left.550^{\circ} \mathrm{C}\right)$ dont $47 \%$ pour la fraction inférieure à $1,25 \mathrm{~mm}$.

— granulométrie : $>11,25 \mathrm{~mm}: 13,47 \% ; 1,25$ à $0,800 \mathrm{~mm}: 2,97 \% ; 0,800$ à $0,500 \mathrm{~mm}: 4,79 \% ; 0,500$ à $0,315 \mathrm{~mm}: 11,36 \% ; 0,315$ à $0,200 \mathrm{~mm}: 11,39 \% ; 0,200$ à $0,125 \mathrm{~mm}: 17,07 \%$; < $0,125 \mathrm{~mm}: 38,95 \%$.

Source hélocrène froide (température maximale en été : $10^{\circ} \mathrm{C}$ ).

1. N.b.: sur 8 nymphes de $P$. paludosa examinées, 2 possédaient 2 cornes thoraciques subégales, de longueur relativement réduite (photo 4). 


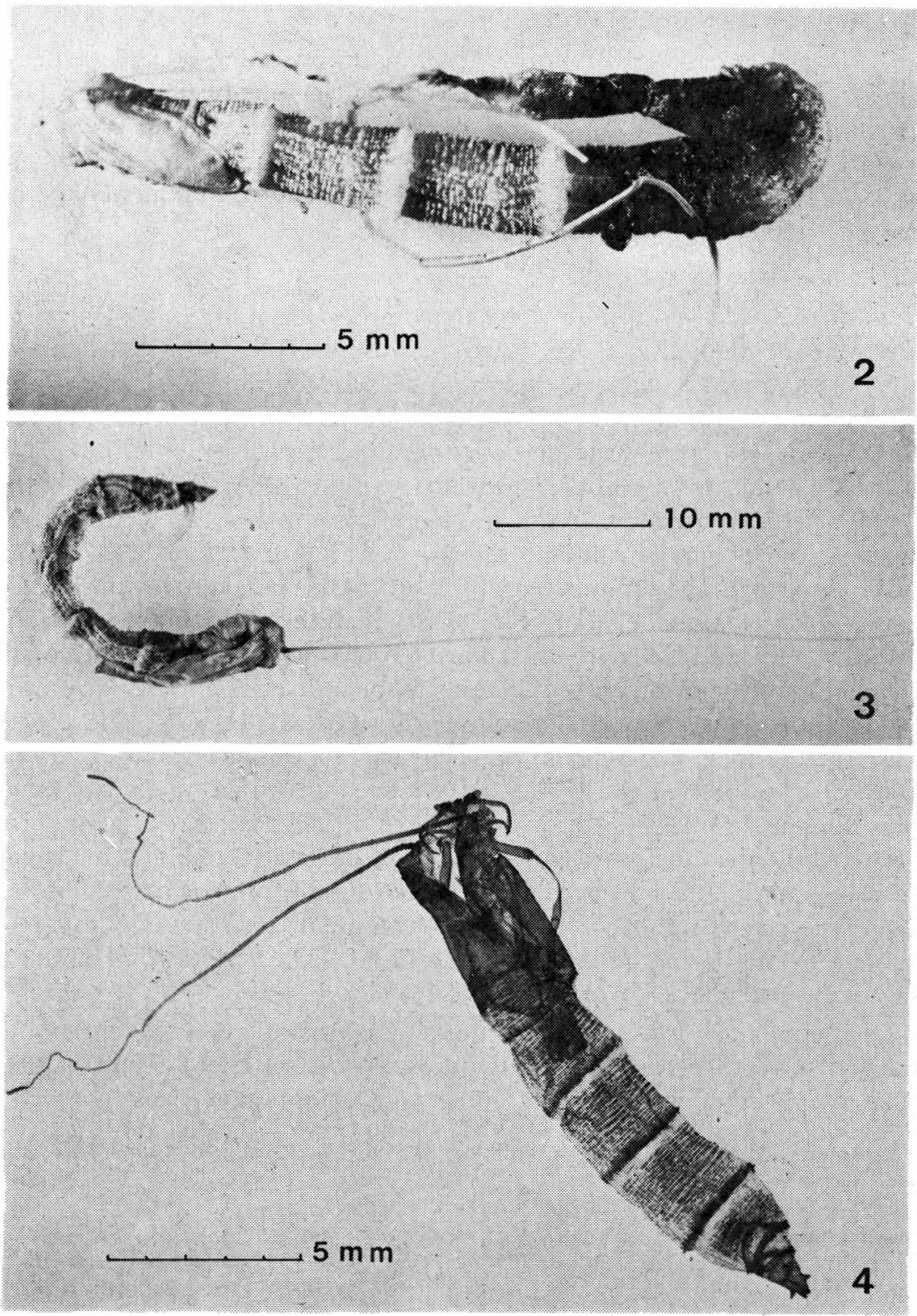

Рното 2. - Nymphe de $P$. albimana se libérant de l'exuvie larvaire.

Рното 3. - Nymphe de $P$. albimana. Noter la longue corne respiratoire antérieure droite et les vestiges du siphon postérieur de la larve pas encore résorbé (nymphe âgée seulement de quelques heures).

Рното 4. - Exuvie nymphale d'un specimen de $P$. paludosa à 2 cornes respiratoires thoraciques subégales. 
Quelques auteurs ont fait des observations approximatives concernant cette durée, le plus souvent sans accorder d'importance à la température et sans même relever de valeurs de cette dernière :

Topsent (1916) : 10 à 12 jours pour $P$. albimana; Alexander (1927) : moins de 5 jours pour $P$. rufocincta; Hodkinson (1973) : 10 à 14 jours pour $P$. lenis, les deux dernières espèces étant nord-américaines. Seul Ameen (1969) a indiqué 8,5 jours environ pour $P$. albimana dans une eau « normally at $19^{\circ} \mathrm{C}$ ».

En fait, la durée de la nymphose des Ptychopteridae est étroitement tributaire de la température ambiante. Chez $P$. albimana, elle peut varier dans des proportions comprises au moins entre 1 et 4 sous la seule action de la température, dans un intervalle de $18^{\circ} \mathrm{C}$.

Expérimentalement, à température constante, on obtient assez aisément une durée d'une semaine à un mois (tableau suivant) :

\begin{tabular}{|c|c|c|c|c|}
\cline { 2 - 5 } \multicolumn{4}{c|}{} & \multicolumn{2}{c|}{ P. albimana } & \multicolumn{2}{l|}{ Pacustris } \\
\hline T. C & Duree J. & N. observations & Duree J. & N. observations \\
\hline $6 / 7$ & 29.31 & 9 & & \\
\hline 10 & 23.25 & 3 & $16,5-17,5$ & 8 \\
\hline $13 *$ & $12,5.15,5$ & 9 & & \\
\hline $15 / 16$ & $8,25.9,5$ & 18 & 8.9 & 12 \\
\hline $20 / 21$ & $6,5.7$ & 9 & 7 & 1 \\
\hline $24 / 25$ & $6.6,25$ & 7 & 6 & 1 \\
\hline
\end{tabular}

* Système de régulation de la température moins précis.

A basse température, la nymphose de $P$. lacustris, espèce de petite taille, est donc sensiblement plus courte que celle de $P$. albimana.

\section{Apparition des nymphes et température}

La température du sédiment où vivent les larves joue un rôle très important.

- a) Dans la nature, lors de prélèvements consécutifs effectués au printemps, on peut constater qu'une élévation rapide (en quelques jours) de la température de plusieurs degrés est immédiatement suivie de l'apparition de nouvelles nymphes, souvent en grand nombre. Ceci est confirmé en élevage à température constante, et plus particulièrement lorsque la température de départ est relativement basse (voisine de $10^{\circ} \mathrm{C}$ ou inférieure). 
Exemple 1:

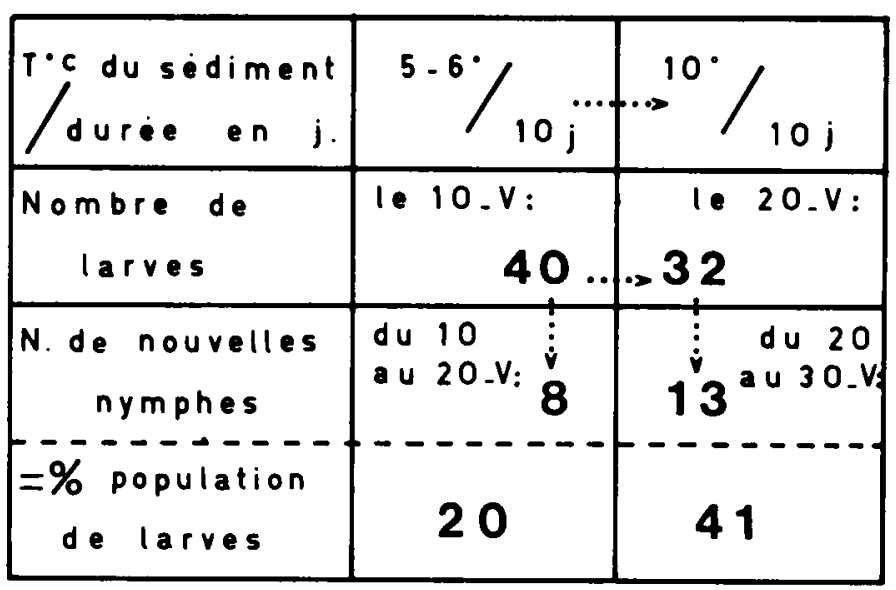

Température du sédiment variable le $10-\mathrm{V}$ selon les points de prélèvement et estimée en moyenne à $7^{\circ} \mathrm{C}$.

Voir également les exemples 2 (29/30-IV) et 3 (117/18-V).

- b) A l'inverse, un abaissement de la température dans un élevage qui produit des nymphes se traduit par un arrêt ou une forte réduction momentané mais immédiats du nombre quotidien des nouvelles exuviations, le plus souvent pendant plusieurs jours.

Exemple 2 : prélèvement de 80 larves de P. albimana.

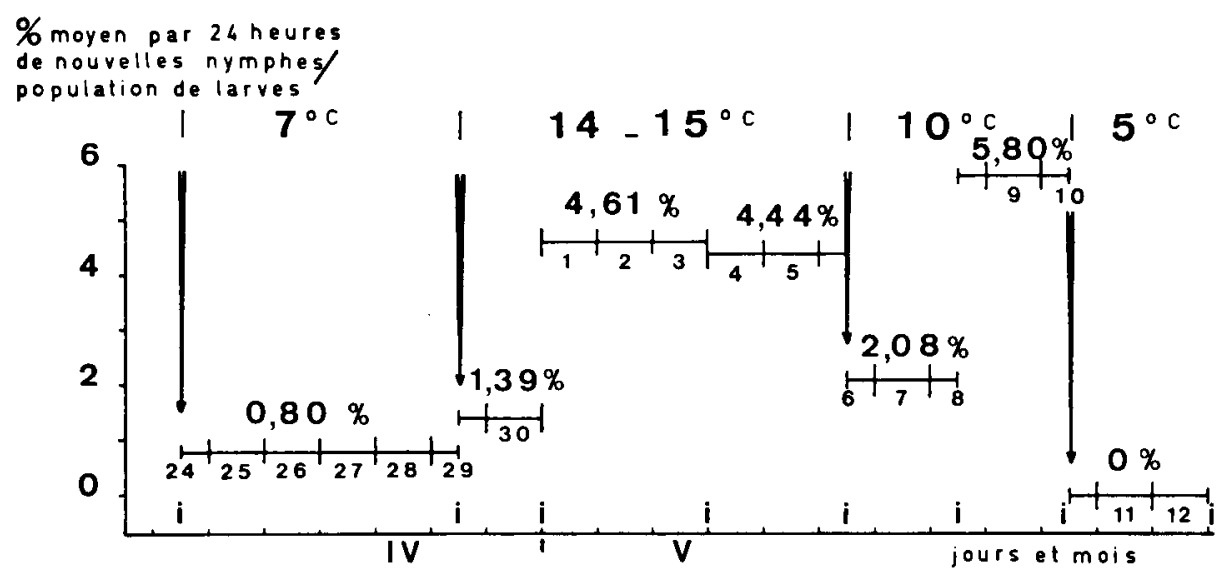

Tri effectué à intervalles irréguliers. i : isolements de nymphes. 
Exemple 3 : prélèvement de 100 larves de $P$. albimana.

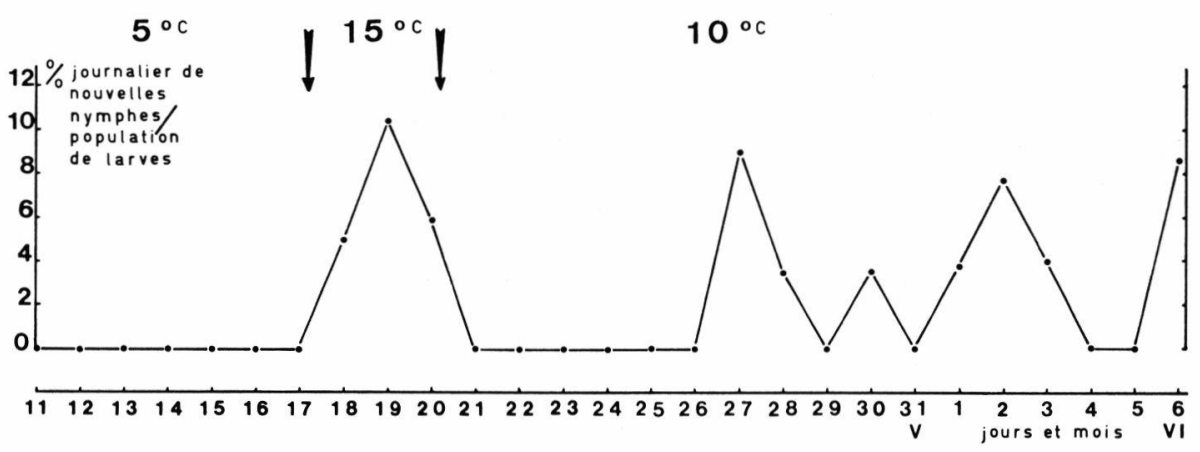

On remarquera la grande rapidité de réponse des Ptychopteridae aux changements de température du milieu, sans doute d'abord par l'action directe de cette dernière sur la longueur de la phase qui précède l'abandon de la dépouille larvaire.

- c) Il est cependant probable que lorsque la période de vol est commencée depuis plusieurs semaines ou mois, $P$. albimana montre une adaptation aux basses températures prolongées. Dans ces conditions défavorables pour la saison, et qui peuvent être considérées comme assez accidentelles, le nombre quotidien des nouvelles exuviations est réduit mais croît avec le temps.

Exemple 4 : prélèvement de 35 larves le $10-\mathrm{V}$ (température du sédiment : voir exemple 1). Elevage à température constante de $7^{\circ} \mathrm{C}$.

\begin{tabular}{|l|c|c|c|}
\hline $\begin{array}{l}\text { Nombres de nymphes } \\
\text { apparues et delai en j. }\end{array}$ & $2 / 71-v$ & $5 / 5$ & $12 / 10$ \\
\hline $\begin{array}{l}\text { \%oyen journalier } \\
\text { par rapport a } \\
\text { la population de larves }\end{array}$ & $<1$ & 3 & 4,5 \\
\hline
\end{tabular}

- d) Il est possible que des nymphes ô se différencient les premières en début de période de vol, lors de l'augmentation rapide de la température maximale des sédiments, due à une photopériode de longueur croissante et un débit faible et en baisse. Les premières nymphes $\hat{o}$ pourraient apparaître quelques jours - ou quelques heures en cas d'amplitudes thermiques journalières importantes - avant les o. Il n'a pu être mis en évidence de différence de longueur entre la nymphose des $\hat{o}$ et celle des o : si la fécondation des premières $q$ est effective, elle implique une protérandrie (voir § II.5). 
Exemple 5 : prélèvement de 18 larves de $P$. albimana le 5-V, dont 17 (8 $\delta, 9$ \% se sont nymphosées ultérieurement comme suit à température croissante :

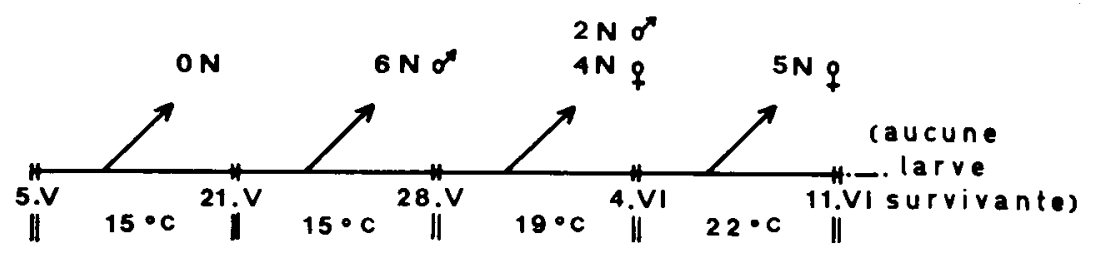

Exemple 6: prélèvement de 15 larves de $P$. lacustris le 26-IV, dont 10 (5 , 5 q) se sont nymphosées ultérieurement comme suit :
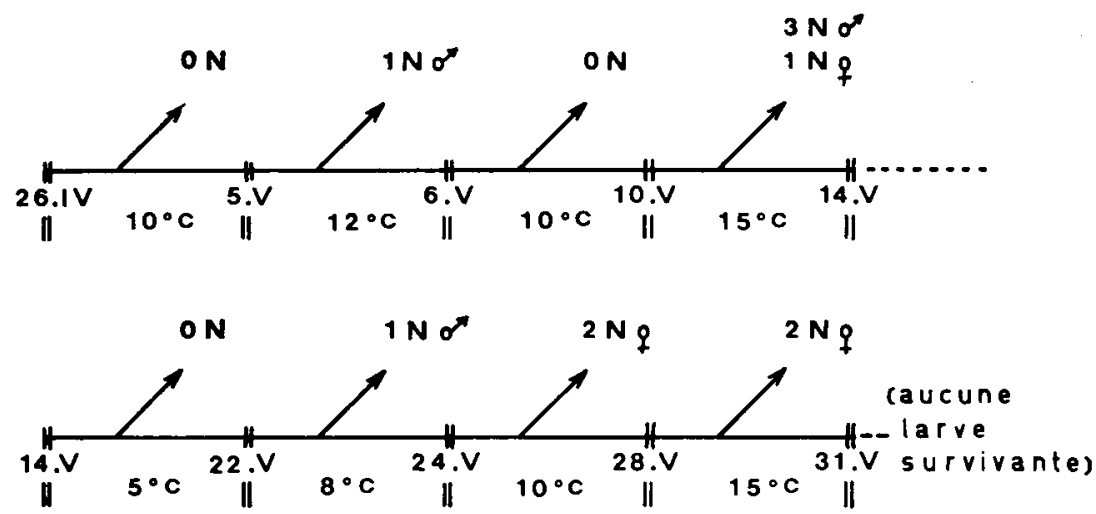

Dans ces deux cas et dans plusieurs autres, des nymphes $q$ sont apparues avec les $\delta$ lors de chaque accroissement brusque de température d'au moins 4 ou $5{ }^{\circ} \mathrm{C}$.

\section{II. - LES MODALITES DE L'ACCOUPLEMENT}

\section{Longévité des imagos}

Accouplements et pontes fécondées ont été observés dans des récipients en matière plastique transparente d'environ 1 litre contenant seulement une compresse de gaze hydrophile fortement imbibée d'eau.

Dans ces conditions qui permettent d'obtenir facilement des larves néonates, les imagos $\delta$ vivent en moyenne plus longtemps que les $q$ dont la longévité n'excède pas trois jours après la première oviposition.

A $20^{\circ} \mathrm{C}$ et en atmosphère très humide, les chiffres suivants ont été relevés : 


\begin{tabular}{l|c|c|c|c|c|} 
& maximum & minimum & moyenne & ecart type & n. observ. \\
\hline$\sigma^{7}$ & $6 \mathrm{j}$ & $2 \mathrm{j} 18 \mathrm{~h}$ & $4 \mathrm{j}$ & $27 \mathrm{~h}$ & 22 \\
\hline 9 & $4 \mathrm{j} 12 \mathrm{~h}$ & $2 \mathrm{j}$ & $3 \mathrm{j} 6 \mathrm{~h}$ & $13 \mathrm{~h}$ & 26 \\
\hline
\end{tabular}

Chez Tipula subnodicornis, les $q$ ont, comparativement aux $\hat{b}$, une mortalité supérieure et une espérance de vie inférieure (Coulson 1962).

\section{Nombre d'accouplements}

Le $\delta$ et la $q$ de $P$. albimana s'accouplent normalement plusieurs fois pendant la vie imaginale : un minimum de deux ou trois fois représente un cas fréquent sur des couples isolés pour observation. Ces accouplements pelıvent être rapprochés (par exemple : 20 minutes, 1 heure) ou beaucoup plus espacés (jusqu'à 3 jours).

Des accouplements peuvent avoir lieu après une ponte.

Exemple : à $15^{\circ} \mathrm{C}, 10^{\circ}$ et 1 \% vus accouplés les $30-\mathrm{V} 197419 \mathrm{~h}, 1-\mathrm{VII} 1 \mathrm{il} \mathrm{h}, 2-\mathrm{VI}$ $10 \mathrm{~h}$ avec une ponte constatée le 11-VII $22 \mathrm{~h}$.

Placés à l'obscurité, les imagos ont tendance à s'accoupler lors de chaque illumination. Ceci a été observé jusqu'à 5 fois de suite sur un même couple.

Exemple : à $15^{\circ} \mathrm{C}, 1 \sigma^{\circ}$ et $1 \%$ accouplés les 1-VI-1974 $22 \mathrm{~h}, 2$-VI $10 \mathrm{~h}, 3$-VI $10 \mathrm{~h}$, 3-VI $19 \mathrm{~h}$ et 4-VI $19 \mathrm{~h}$ avec une ponte observóe le 3-VI $10 \mathrm{~h}$.

Linley et Hinds (1974) ont montré que les ồ du Nématocère Culicoides melleus (Ceratopogonidae) étaient fertiles jusqu'à 6 ou 7 accouplements. Rogers (1937) a cependant remarqué chez plusieurs espèces de Tipulidae $s$. $l$. une fertilité fortement diminuée après le premier accouplement.

\section{Durée de l'accouplement}

L'accouplement proprement dit (préliminaires non compris) a une durée assez variable, souvent de l'ordre de 10 à 20 minutes. Cette durée diminue avec une élévation de la température ambiante:

$-15 / 16^{\circ} \mathrm{C}$ : 9 à 31 minutes; moyenne : 18,5 (5 mesures), écart-type : 8.

- 20/22 ${ }^{\circ} \mathrm{C}: 5,5$ à 19 minutes; moyenne : 11 ( 9 mesures), écart-type : 4.

A $10^{\circ} \mathrm{C}$, on peut encore observer des tentatives d'accouplement, cependant très maladroites. A une température inférieure, les imagos paraissent incapables de s'accoupler. 


\section{Position des conjoints}

Les premiers signes d'excitation sexuelle chez le $\hat{a}$ apparaissent souvent après le contact, même fortuit, entre les pattes des deux spécimens. La paire de pattes antérieures du $\hat{o}$ paraît jouer un rôle primordial - sans doute du fait d'organes sensoriels (Séguy 1950) et il n'a pu être observé d'accouplements lorsque les ô sont privés de leurs tarses I. Or, l'accouplement a lieu après amputation des tarses II ou III.

Le $a$ se tient au-dessus de la $q$, légèrement en retrait et dans le même sens qu'elle. Son abdomen est recourbé sur la face dorsale, la pince génitale dirigée vers l'avant. Les deux abdomens sont croisés verticalement et la partie distale de celui du ô est située sous celui de la .. La prise de la pince génitale a lieu dans cette attitude après un délai qui peut varier de quelques secondes à plus de 4 minutes. La position définitive est alors obtenue rapidement - en moins de 10 secondes - par un mouvement de 180 degrés du $\delta$ dont l'abdomen va se trouver dans le prolongement de celui de la $q$. Les deux conjoints sont opposés, ce qui a été noté par Topsent (1916).

Sur substrat vertical, cas le plus fréquent, la $\%$ a toujours été observée en position haute. Sur substrat horizontal, elle entraîne le plus souvent le $\hat{\delta}$ à sa suite vers une surface d'appui verticale.

En fin d'accouplement, avant séparation, les ailes du ô vibrent à plusieurs reprises et les deux conjoints peuvent toucher - la $\&$ en particulier - la région distale des deux abdomens avec les tibias postérieurs.

\section{Age des imagos lors de l'accouplement}

\section{- 9 .}

Les $q$ attirent d'autant plus les $\delta$ que leur émergence est récente et donc que leur abdomen est encore mou et plus ou moins distendu. Au laboratoire, c'est d'ailleurs dans ce cas que les pontes de $P$. albimana ont le plus de chances d'être fécondées dans de fortes proportions. A l'inverse, aucun accouplement n'a été noté avec des $q$ âgées de plus de 3,5 jours. Sur le terrain, il est fréquent de voir plusieurs $\delta$ se presser fébrilement autour d'une o encore en cours d'exuviation. En général, l'un d'eux se superpose à la $\%$ peu après l'apparition du thorax de celle-ci. L'accouplement a lieu dès que l'abdomen est libéré de l'exuvie nymphale, alors même que les ailes ne sont pas encore complètement déplissées.

Traynier et Burton (1970) ont remarqué que l'intérêt du ô de Tipula paludosa pour la $\circ$ ne se manifeste que très près de celle-ci $(1 \mathrm{~cm})$ et d'autre part que c'est la région antérieure de la $q$, et non son abdomen, qui attire le ${ }^{\hat{}}$. Ces constatations paraissent applicables à $P$. albimana. 
D'autre part, Coulson (1962) a noté que la fécondation des $q$ de Tipula paludosa et de $T$. subnodicornis a lieu dès leur émergence. Giard (1895) et Kirk (1923, rapporté par Séguy 1950) ont fait des observations analogues, respectivement sur un Tipula et un Culicidae.

- $\hat{\text { ô. }}$.

L'âge du $\delta$ au moment de l'accouplement (dans le cas de copulalations toujours suivies de ponte et d'éclosion) a été compris entre un minimum de 2 à 3 heures et un maximum de 5,5 jours, pour une dizaine d'observations. Le ô fraîchement émergé, même actif, paraît incapable de s'accoupler.

Ces particularités vont dans le sens d'une éventuelle protérandrie en début de période de vol (voir § I.3.d).

\section{Temps écoulé entre accouplement et ponte}

Le minimum observé a été de $10 \mathrm{~h}$, le maximum de $32 \mathrm{~h}$, pour une quinzaine d'observations. Coulson (1962) a relevé $7 \mathrm{~h}$ chez Tipula subnodicornis et $17 \mathrm{~h}$ chez $T$. paludosa.

\section{TRAVAUX CITES}

Alexander (C. P.). 1927. - Diptera Fam. Ptychopteridae. Genera Insectorum, $188: 11-12+1 \mathrm{pl}$.

AMEen (M. ul.). 1969. - Metamorphosis of some of the organ systems in the fly Ptychoptera albimana F. Trans. $R$. ent. Soc. Lond., 121 (6) : 235-279.

ANGELIER (E.). 1953. - Recherches écologiques et biogéographiques sur la faune des sables submergés. Archs Zool. exp. gén., 90 (2):37-161.

Coulson (J. C.). 1962. - The biology of Tipula subnodicornis Zetterstedt, with comparative observations on Tipula paludosa Meigen. J. Anim. Ecol., 31 : 121.

Freeman (P.). 1950. - Family Ptychopteridae. In : Handbooks for the Identification of British Insects. R. ent. Soc. Lond., 9 (2) : $73-76$.

Giard (A.). 1895. - Note sur l'accouplement de Tipula rufina Meig. (Dipt.). Bull. Soc. ent. Fr., séance du 10-IV-1895: cxcI-cxcII.

HodKINSON (I. D.). 1973. - The immature stages of Ptychoptera lenis lenis (Diptera, Ptychopteridae) with notes on their biology. Can. Ent., $105: 1091$ 1099.

Joost (W.). 1974. - Ptychoptera peusi n. sp. - eine neue Faltenmücke (Dipt., Ptychopteridae) aus dem oberen Baksangebiet (USSR, Zentralkaukasus). Ent. Nachr., 18 (11-12): 173-178.

KIRK (H. B.). 1923. - Notes on the mating-habits and early life-history of the Culicid Opifex fuscus Hutton. Trans. N. Z. Inst., $54: 400-406$.

Linley (J. R.) et Hinds (M. J.). 1974. - Male potency in Culicoides melleus (Coq.) (Diptera, Ceratopogonidae). Bull. ent. Res., 64 : 123-128.

Peus (F.). 1958. - Liriopeidae. In Lindner E.: Die Fliegen der Palaearktischen Region. $10 \mathrm{~b}: 10-44$.

Rogers (J. S.). 1957. - Craneflies. In Needham et coll. : Culture methods for invertebrate animals. $2^{e}$ édition, 1959: Dover, New York: $590 \mathrm{p}$.

SÉGUY (E.). 1950. - La Biologie des Diptères. Encyclopédie Entomologique, 26. Lechevalier, Paris : 609 p. 
Seligo (A.). 1928. - Zur Kenntnis der Mückengattung Liriope Mg. Ber. westpreuss. bot.-zool. Ver., $50: 231-247+4 \mathrm{pl}$.

StubBS (A. E.). 1972. - A review of Information on the Distribution of the British Species of Ptychoptera (Dipt.: Ptychopteridae). Entomologist, 105 : 23-38.

Thomas (A.). 1968. - Limoniidae et Ptychopteridae du Sud-Ouest de la France (Diptera) (1 ${ }^{\circ}$ note). Annls Limnol., 4 (2) : 225-234.

Thомas (A.). 1969. - Sur l'importance des Diptères dans l'environnement de quelques cours d'eau des Pyrénées. Annls Limnol., 5 (1) : $61-71$.

Thomas (A. G. B.). 1977. - Limoniidae et Ptychopteridae du Sud-Ouest de la France (Diptera, Nematocera) ( ${ }^{\circ}$ note). Annls Limnol., 13 (1) : 47-55.

Tonnoir (A.). 1919. - Notes sur les Ptychopteridae (Dipt.). Description d'espèces nouvelles et d'un organe sexuel auxiliaire chez certains mâles. Annls Soc. ent. Belg., 59 : 115-122.

TOPSENT (E.). 1916. - Etude sur Ptychoptera albimana (Diptère, Némocère). Archs Zool. exp. gén., 55 (5) : 81-94.

Traynier (R. M. M.) et Burtan (D. J.). 1970. - Mâle response to females in the marsh crane fly, Tipula paludosa Mg. (Diptera, Tipulidae). J. ent. Soc. Brit. Columbia, $67: 21-22$.

ZiteK-ZWYRTEK (K.). 1971. - Czechoslovak species of the family Ptychopteridae (Diptera). Acta ent. bohemoslov., $68: 416-426$. 